\title{
First Measurements of Jet Substructure in ATLAS
}

\author{
Sarah Livermore ${ }^{1}$ \\ On behalf of the ATLAS collaboration \\ University of $O x$ ford \\ E-mail: sarah. Iivermoredphysics.ox.ac.uk
}

\begin{abstract}
We present a summary of studies on the substructure of jets collected by the ATLAS detector in $35 \mathrm{pb}^{-1}$ of proton-proton collisions at $7 \mathrm{TeV}$ centre of mass energy. At these energies, electroweak scale objects are produced with large transverse momenta, resulting in their decay products being localised in a relatively small region of the detector. Substructure variables have a powerful potential for distinguishing the signatures of these decays from those of the QCD background with the caveat that the substructure generated by QCD radiation is well understood. Therefore, we show the comparison between data and prediction for jet mass and a key substructure variable, from an inclusive sample of high- $\mathrm{p}_{\mathrm{T}}$ jets in the above dataset. Good agreement is seen. The use of this strategy in the search for massive new particles via their decays into top quark pairs is also summarised.
\end{abstract}

The 2011 Europhysics Conference on High Energy Physics-HEP2011 Grenoble, Rhône-Alpes

July 21-27 2011

\footnotetext{
1 Presenter
} 


\section{Introduction}

At the LHC, particles can be produced with significant Lorentz boosts, resulting in collimated decay products. A study of the jet mass and internal substructure can help to understand the processes which initiated the decay. This can increase the search potential in many analyses by reducing the large background from QCD. It is particularly applicable to searches for high $\mathrm{p}_{\mathrm{T}}$ "boosted" top pair production by massive new particles.

\section{Jet formation and substructure}

Jet algorithms combine clusters of calorimeter energy deposits to form jets with a pre-defined radius, $R$, in $\eta-\varphi$ space. Two such algorithms have been employed for these studies: anti- $k_{T}$ [1] (with $\mathrm{R}=1.0$ ) and Cambridge-Aachen [2] (with $\mathrm{R}=1.2$ ). In this study, jets formed with the latter also underwent a process called filtering to remove contamination from the underlying event.

The jet mass is defined as the sum of the 4-vectors of all constituent clusters and is a powerful variable for identification of the parent particle. The splitting scale is the energy at which the jet splits into two and provides the ability to distinguish heavy particle decays from asymmetric QCD splittings.

\section{Understanding jet mass and substructure}

A sample of inclusive jets was collected from $35 \mathrm{pb}^{-1}$ of proton-proton collisions at a centre of mass energy of $7 \mathrm{TeV}$ [3]. Predictions from several leading order parton shower Monte Carlo generators were found to be in agreement with data, as shown in figure 1, indicating that jet substructure generated by QCD radiation is well understood.
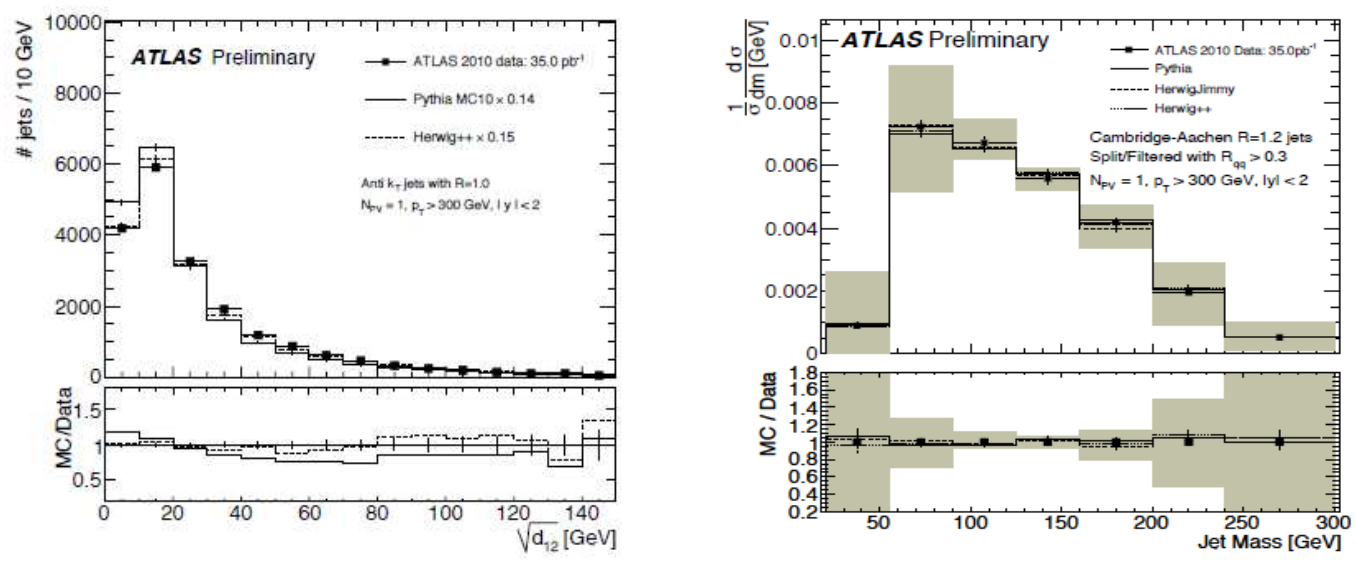

Figure 1: Left - distribution of the splitting scale of anti- $\mathrm{k}_{\mathrm{T}}$ jets. Right - invariant mass spectrum of Cambridge-Aachen jets, corrected for detector effects. 


\subsection{Systematic uncertainties}

An estimation of the systematic uncertainty associated with these measurements was performed by comparing jets reconstructed using energy deposits in the calorimeter and using tracks in the inner detector. These sub-detectors have largely uncorrelated systematic effects. The uncertainty on the jet mass scale was determined to be 6-7\%, dependent on jet type.

\subsection{The effect of multiple proton-proton interactions}

The results presented above are based on events with exactly one primary vertex, and hence the effects of multiple interactions, or pile-up, are greatly reduced. The mass of these larger jets is found to be correlated with the amount of pile-up activity [3]. The use of grooming techniques (e.g. filtering) to reduce this dependency is being investigated.

\section{Boosted top identification}

Conventional searches for top quarks aim to resolve their hadronic decay products using jets of size $\mathrm{R}=0.4$. In the high $\mathrm{p}_{\mathrm{T}}$ regime, a large $(\mathrm{R}=1.0)$ jet is used to contain all the decay products, rather than attempting to identify the individual jets. Figure 2 shows simulated distributions of jet mass and substructure for QCD and top jets. These are important inputs into the ttbar resonance search due to their power of discrimination between the two types of jets [4].
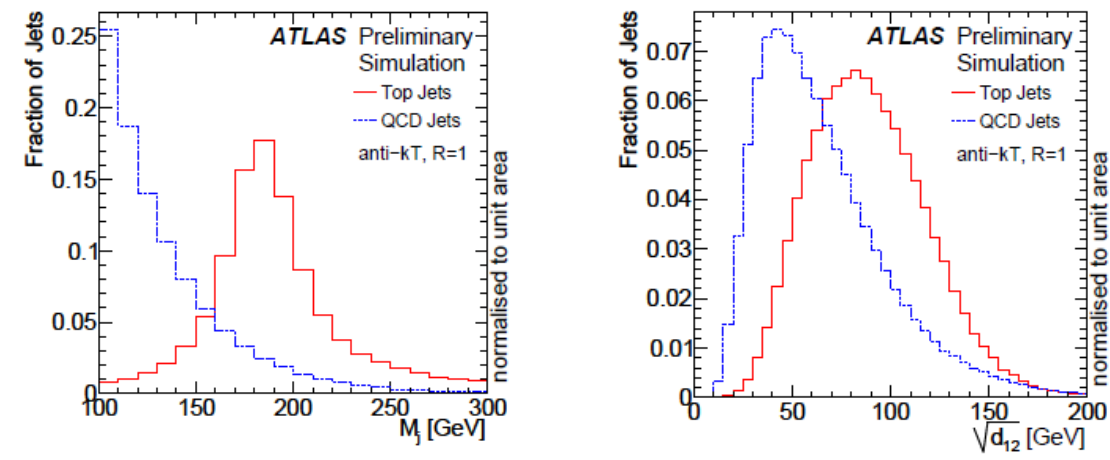

Figure 2: Jet mass (left) and splitting scale distributions (right) for anti- $\mathrm{k}_{\mathrm{T}} 1.0$ jets initiated by top quark decays and QCD processes.

\section{References}

[1] M. Cacciari, G. P. Salam, and G. Soyez, The Anti- $k_{T}$ Jet Clustering Algorithm, JHEP 04 (2008) 063 , arXiv:0802.1189 [hep-ph]

[2] Y. L. Dokshitzer, G. D. Leder, S. Moretti, and B. R. Webber, Better Jet Clustering Algorithms, JHEP 08 (1997) 001, arXiv:hep-ph/9707323

[3] ATLAS Collaboration, Measurement of Jet Mass and Substructure for Inclusive Jets in 7 TeV pp Collisions with the ATLAS Experiment, Tech. Rep. ATLAS-CONF-2011-073, CERN, Geneva, 2011

[4] ATLAS Collaboration, Prospects for Early ttbar Resonance Searches in ATLAS, Tech. Rep. ATL-PHYS-PUB-2010-008, CERN, Geneva, 2011 